\title{
EVOLUTION OF MASSIVE STARS THROUGH LATE EVOLUTIONARY PHASES
}

\author{
Susan A. Lamb
}

University of California, Los Angeles

\section{INTRODUCTION}

The theoretical study of the evolution of massive stars has considerable relevance to many branches of observational astronomy. As an example, one may mention, first, the direct application of theoretical models in interpreting the observed properties of supergiants, and second, the use of calculations of the nucleosynthesis taking place in these stars, together with observed supergiant mass loss rates and supernova calculations, to estimate the rate and type of nucleosynthetic enrichment of the interstellar medium by supergiants.

In this brief review of the evolution of massive stars, I shall emphasize theoretical calculations of the later evolutionary phases, namely those beyond core helium burning, and their observational implications, although I shall make some reference to the earlier stages where necessary.

In Section 2 I discuss nucleosynthesis in massive stars and its observable consequences. The interpretation of the ratio of blue to red supergiants is considered in section 3, and in Section 4, I briefly discuss the observed minimum absolute magnitude of red supergiants, and the possibility that the highest mass Cepheids observed are massive stars in late evolutionary phases. Section 5 contains a summary. 


\section{NUCLEOSYNTHESIS IN MASSIVE STARS}

\section{I Theoretical Calculations}

The most extensive nonexplosive nucleosynthesis takes place in those stars that are sufficiently massive to burn carbon quiescently. The lower mass limit to this class of stars is approximately 9 or $10 \mathrm{M}_{\odot}$; the lack of precision reflects the
uncertainty in the rate of the carbon burning reaction. The products of carbon burning are later reprocessed through a series of nuclear burning phases which eventually produce the "iron peak" elements, but none of these nuclear reactions ignite in a sufficiently degenerate environment with enough energy release to explode the star. Quasi-static evolution is terminated when the core of the star becomes unstable due to the production of electron-positron pairs, or photodissociation of the 'iron peak' nuclei into $\alpha$-particles. The former instability sets in prior to the latter only in very massive stars $(\mathrm{M} \gtrsim 80-100 \mathrm{M}$, see Arnett, 1972) and, therefore, stars with initial masses in the range $9-10<\mathrm{M} / \mathrm{M} \lesssim 80-100$ progress through the full range of possible nuclear burning stages. As stars in this mass range are very much more numerous than those with $\mathrm{M}>80 \mathrm{M}$, and potentially produce a wider range of nuclei in their interiors, they have been the subject of most nucleosynthetic studies.

Many authors have investigated the detailed structural evolution and the associated chemical evolution of massive stars through the phases of core hydrogen and core helium burning, but only relatively recently has there been detailed investigation of the later nuclear burning phases, that is, from core carbon burning onwards. Many of the calculations have been for stellar cores devoid of envelopes, where the focus of interest has been the nucleosynthesis that takes place in the deep interior. Thus Rakavy et al. (1967) and Ikeuchi et al. (1971, 1972) have evolved a series of carbon-oxygen cores from before carbon ignition to the onset of instability, while Sugimoto (1970) has followed helium cores from core helium burning through core oxygen burning; these latter models have been carried through to the onset of instability by Sugimoto and Nomoto (1974, and references therein). Arnett (1977, and references therein) has made an extensive study of helium cores, evolving them through Si-burning. Ignoring the hydrogen envelopes of massive stars is a reasonable way to explore the detailed nucleosynthesis in the core, assuming that envelope convection does not penetrate through the base of the hydrogen envelope, but no information on observable surface parameters is obtained.

Early investigations of the late evolutionary stages of complete massive stars were made by Hayashi and Cameron (1962) and by Stothers and Chin (1969). More recently, Endal (1975) 
has evolved a $15 \mathrm{M}$ star from the main sequence to neon ignition in the core, while Lamb et al. (1976) have evolved $15 \mathrm{M}$ and $25 \mathrm{M}$ stars from the main sequence through core carbon burning. The latter work includes evolutionary tracks in the theoretical Hertzsprung-Russell diagram.

The results of nucleosynthesis are sensitive to the physical conditions in the star, that is, the run of temperature and density with radius, and the details of energy transport. In the cores of massive stars these quantities are determined, from core carbon burning onwards, predominantly by the loss of energy due to neutrino emission. 'Pair', 'plasma', and 'photo' neutrino processes dominate (see Beaudet et al., 1967) whereas

bremstrahlung neutrinos (see Festa and Ruderman, 1969) make only a small contribution. The final distribution of nuclei through the stellar core depends sensitively on the extent in mass of the convective core formed during each successive nuclear burning phase. A sequence of convective regions diminishing in mass as evolution progresses is required to produce a layering of regions with different mean molecular weights, that is, a wide variety of nuclei, in the core. The size of the convective core is determined by the rates and location of neutrino energy losses (Arnett, 1972, and Lamb et al., 1976). As the central temperature of the star increases, these energy loss rates approach the nuclear energy generation rates, ensuring smaller convective cores. The calculations of Arnett (1977, and references therein), which do include neutrino energy losses, yield a stratified stellar core with the unburned helium surrounding successive layers composed largely of carbon, oxygen, neon, magnesium, silicon, and iron, as one proceeds inward. The precise mass and composition of each layer depends on the mass of the star; tables of the final core composition as a function of mass are given by Arnett (1978).

Although the major burning stages of stellar evolution only produce nuclei with $A \hat{\imath} 60$, nuclei of much higher atomic weight can be produced during these phases by neutron captures on the lighter nuclei. Interesting quantities of neutrons are produced in the core during the later part of core helium burning, and the resulting nucleosynthesis has been investigated by Couch et al. (1974) and Lamb et al. (1977). It should be noted that the nuclei produced in this way are contained wholly within the helium convective core. Many will not survive the later evolution but will act as seed nuclei for further processing. A very detailed calculation of the composition of a massive star nearing the end of its quasi-static evolution has not yet been performed.

\subsection{Observational Implications}

A surface composition displaying products of nuclear burning 
can occur in supergiants if these products are transported upwards through the envelope to the surface or if the outer layers are shed, thereby revealing inner regions which have undergone nuclear processing. Early-type supergiants do not possess convective envelopes. Thus stars of this class which display evolved surface compositions are most likely to have undergone considerable mass loss (see Walborn, 1976, and Dearborn and Eggleton, 1977) or to have experienced some mixing process such as meridianal mixing. On the other hand, the rapid drop in surface temperature which occurs as a supergiant crosses the Hertzsprung gap towards the red is accompanied by the development of a convective envelope. This dredges up material which has been partially burned during core hydrogen burning earlier, and deposited outside the retreating convective core. The ratio of nitrogen to hydrogen in the envelope is raised to about five times its initial value by this process (see Lamb et al., 1976, for details). At no time during the major fraction of the life of these massive stars does it appear that the envelope convection penetrates through the hydrogen burning shell. Thus the products of helium and later burning stages are not expected to be brought to the stellar surface by convective transport. Mass loss of the entire hydrogen and some of the helium envelope is required to reveal these burning products.

If supergiants end their quasi-static evolution as supernovae, it may be possible to detect the products of nuclear processing in young supernova remnants which have not yet become mixed with large masses of interstellar medium. The supernova remnants Cas A, Pup A, and Kepler are possible examples. Studies of Cas A by Peimbert and van den Bergh (1971), Peimbert (1971), and Chevalier and Kirshner (1978) reveal quite different products of nuclear processing in different parts of the remnant. An interpretation of these abundances in terms of the evolutionary history of the progenitor has been suggested by Lamb (1978). In general, young supernova remnants may display the nucleosynthetic products of several stages of core nucleosynthesis, if the progenitor has undergone substantial mass loss prior to the supernova explosion.

\section{THE RATIO OF BLUE TO RED SUPERGIANTS}

The observed ratio of blue to red supergiants is of considerable interest, as theoretical calculations have shown that this ratio is very sensitive to the neutrino energy loss rate. This has led several authors (Stothers, 1969, and references therein) to attempt to confirm or refute the existence of the universal Fermi interaction, by a detailed comparison of observation and theory. However, other uncertainties in the calculations are known to effect this ratio, for example, the choice of convective 
stability criterion (see Chiosi and Summa, 1970) and the amount of mass loss during the early evolution (Hartwick, 1967, de Loore et al., 1977, and Chiosi et al., 1977). Further, it has also been pointed out that interpreting the ratio of blue to red supergiants as a measure of the ratio of evolutionary timescales of single massive stars through different evolutionary phases is erroneous. Thus, for example, Choisi and Summa (1970) demonstrated that the presence of binary systems among massive stars increases the observed blue to red ratio by an unknown amount, while Ferrari et al. (1970) argued that the sample of red supergiants used previously is contaminated by lower mass stars, which have evolved into the red supergiant region, thereby decreasing the blue to red ratio. The spread in the time of formation of stars in any stellar association, or collection of associations, used to determine this ratio will necessarily introduce the latter uncertainty (Lamb et al., 1976). Thus it would appear that the observed ratio of blue to red supergiants is a poor tool for probing basic physics, but is, nevertheless, an observational quantity which must eventually be given a full theoretical explanation.

\section{RED SUPERGIANTS AND MASSIVE CEPHEIDS}

Observations of stellar associations and galactic clusters (cf. Humphreys, 1970, and this volume, p. 263) indicate that red supergiants have a minimum $\mathrm{Mbl}_{\mathrm{bol}} \sim-9$. This bolometric magnitude corresponds to the luminosity expected for a star of mass slightly greater than $25 \mathrm{M}$, during its later evolution, and the question then arises of the fate of the still more massive stars. One possibility for preventing the most massive stars from reaching the red supergiant region is to invoke substantial mass loss during the early evolution, that is, during core hydrogen and core helium burning, when the stars are 0 and $B$ supergiants. Hartwick (1967) was the first to demonstrate that substantial mass loss can take the evolutionary tracks back towards the blue. On the other hand, the possibility that stars of about $25 \mathrm{M}_{0}$ and greater may not evolve into the red supergiant region, even ${ }^{\odot}$ without mass loss, until very late in their evolution, if at all, has emerged from recent calculations by Lamb et al. (1976). They find that a $25 \mathrm{M}_{\Theta}$ star stays to the blue of $\overline{\mathrm{log}_{10}}\left(\mathrm{~T}_{\mathrm{S}}\right) \sim 3.9$ throughout its evolution up to at least the end of core carbon burning, that is, to very near the end of its observable life. By contrast, they find that a $15 \mathrm{M}$ star evolves across the Hertzsprung gap, and burns carbon âs a red supergiant with $\log _{10}\left(T_{S}\right) \sim 4.5$. The different surface temperatures of these two stars reflect substantially different envelope structures, the $25 \mathrm{M}_{\odot}$ star having no convective envelope.

Turning now to a consideration of long period Cepheids, we 
note that the longest period Cepheids observed by Gascoigne (1969) have periods $~ 130$ days, which indicate stars of about $20 \mathrm{M}$. It has been suggested by Lamb et al. (1976) that these Cepheids are in the core carbon burning phase of evolution, and occur at the intersection of the instability strip and the core carbon burning band. However, the evolution after the carbon burning phase is so rapid that the stellar envelope has no time to adjust to changes taking place in the core. Thus, if a star becomes a Cepheid during core carbon burning it will stay one until its demise. Chiosi et al. (1977) have also pointed out that stars which become Cepheids need not have had an initial mass of $20 \mathrm{M}$ or less, if appreciable mass loss occurred during their early 巳̊volution.

\section{SUMMARY}

In this brief review of some aspects of the late evolution of massive stars I have discussed, 1) the theoretical calculations of the evolution, placing particular emphasis on the factors influencing nucleosynthesis, 2) possibilities for observing the products of this nucleosynthesis, at the surface of stars, and in young supernova remnants, 3) the interpretation of the ratio of blue to red supergiants, 4) the observed maximum luminosity limit for red supergiants, and 5) the possibility that the longest period Cepheids are massive stars in late evolutionary phases.

I wish to thank Dave Arnett, Icko Iben, Jim Truran, and Roger Ulrich for interesting and helpful discussions on different aspects of the material presented in this paper, and the Aspen Center for Physics for hospitality.

\section{REFERENCES}

Arnett, W.D. (1972). Astrophys. J. 176, 699.

Arnett, W.D. (1977). Astrophys.J. Suppl. 35, 127.

Arnett, W.D. (1978). Astrophys. J. (in press).

Beaudet, G., Petrosian, V. and Salpeter, E.E. (1967). Astrophys. J. $150,979$.

Chevalier, R.A. and Kirschner, R.P. (1978). Astrophys. J. (in press).

Chiosi, C., Nasi, E. and Sreenivasan, S.R. (1977). Astron. Astrophys. (in press).

Chiosi, C. and Summa, C. (1970). Astrophys. Space Sci. 8, 478.

Couch, R.G., Schmeidekamp, A.B. and Arnett, W.D. (1974). Astrophys. J. 190, 95.

Endal, A.S. (1975). Astrophys. J. 197, 405.

Ferrari, A., Galeotti, P., Silvestro, G. and Trussoni, E. (1970). Astrophys. Space Sci. 9, 181. 
Festa, G.G. and Ruderman, M.A. (1969). Phys. Rev. 180, 1227. Gascoigne, S.C.B. (1969). Mon. Not. Roy. Astron. Soc. 146, 1. Hartwick, F.D.A. (1967). Astrophys. J. 150, 953.

Hayashi, C. and Cameron, R.C. (1962). Astrophys. J. 136, 166. Humphreys, R.M. (1970). Astrophys. Letters 6, 1.

Ikeuchi, S., Nakazawa, K., Murai, T., Hoshi, R. and Hayashi, C. (1971). Prog. Theor. Phys. Kyoto 46, 1713. (1972). ibid. 48, 1870.

Lamb, S.A. (1978). Astrophys. J. (in press).

Lamb, S.A., Howard, W.M., Truran, J.W. and Iben, I. (1977). Astrophys. J. 217, 213.

Lamb, S.A., Iben, I. and Howard, W.M. (1976). Astrophys. J. $207,209$.

de Loore, C., De Greve, J.P. and Lamers, H.J.G.L.M. (1977). (preprint).

Peimbert, M. (1971). Astrophys. J. 170, 261.

Peimbert, M. and van den Bergh, S. (1971). Astrophys. J. 167, 223.

Rakavy, G., Shaviv, G. and Zinamon, Z. (1967). Astrophys. J. $150,131$.

Stothers, R. (1969). Astrophys. J. 155, 935.

Stothers, R. and Chin C.-W. (1969). Astrophys. J. 158, 1039.

Sugimoto, D. (1970). Prog. Theor. Phys. Kyoto 44, 599.

Sugimoto, D. and Nomoto, K. (1974). In IAU Symposium 66, Late Stages of Stellar Evolution, R.J. Tayler and J. Hesser, eds., Reidel, Dordrecht, p. 105.

Walborn, N. (1976). Astrophys. J. 205, 419. 


\section{DISCUSSION}

TAYLER: I hope you'll excuse me while I congratulate the last two speakers on their results and also make a historical reminiscense. About four years before Henry Norris Russell died I was evolving stars - I haven't, since then, evolved any stars - and I evolved a massive star towards the onset of hydrogen exhaustion, using a desk calculator. I calculated that it would take me 20 months, working 6 hours a day, five days a week to evolve a one solar mass star - and that is why I gave up stellar evolution at that moment. I think that the results shown by Allen Sweigart and by Susan Lamb have shown us how far things have come in these last 20 - odd years.

BIDELMAN: Could you say a word about the evolution of stars of somewhat smaller mass, say 5 to $10 \mathrm{M}_{\odot}$ ?

LAMB: Stars in this mass range are thought to end their evolution in carbon-detonation supernova, although if all of these stars exploded in this way it would lead to an overproduction of carbon. Various possible mechanisms for avoiding the explosion or reducing the mass range of stars involved in this type of supernova have been investigated, for example, the Urca process and substantial mass loss. The end points of evolution of these stars are not well studied partly because the evolutionary calculations are severely complicated by the occurence of helium shell flashes. These latter have been extensively studied by Iben.

COX: What is the maximum mass that you believe has a blue loop?

LAMB: I think that it would be somewhere between 10 and $13 \mathrm{M} \odot$, perhaps. But that's an uncertain area. 